\title{
Drogas: famílias que protegem e que expõem adolescentes ao risco
}

\section{Drugs: families that protect and that expose teenagers to risk}

\author{
Rogério Lessa Horta, Bernardo Lessa Horta e Ricardo Tavares Pinheiro
}

\begin{abstract}
Resumo
Objetivo: Este estudo tem por objetivo avaliar o consumo de substâncias psicoativas (bebidas alcoólicas, tabaco e drogas ilícitas) por adolescentes do município de Pelotas (RS), de acordo com a presença de pai e/ou mãe no domicílio e o hábito de fumar ou não de ambos. Métodos: Foi realizado, em 2002, um estudo transversal na área urbana de Pelotas. Empregou-se amostragem em múltiplos estágios para se obter uma amostra de adolescentes entre 15 e 18 anos de idade. As entrevistas foram realizadas com questionário auto-aplicado. Resultados: A coabitação de pais ou mães e adolescentes parece reduzir significativamente as chances de os adolescentes consumirem tabaco, diminuir discretamente para drogas ilícitas e não tem influência em relação ao consumo de bebidas alcoólicas. Conclusão: 0 tabagismo de pais e mães parece aumentar as chances de os adolescentes fumarem. Não houve interação entre as duas variáveis em relação ao consumo de qualquer das substâncias estudadas.
\end{abstract}

Palavras-chave: tabagismo, família, álcool, drogas.

\begin{abstract}
Objective: This study was aimed to assess the relationship between substance use (alcoholic beverages, smoking and drug use) among teenagers and cohabitation with parents, as well as the relationship between substance use and parental smoking. Methods: In 2002, a cross-sectional survey was carried out in the urban area of Pelotas, southern Brazil. Multi-stage sampling was used to obtain a sample of adolescents aged between 15 and 18 years. Adolescents were interviewed using a self-applied confidential questionnaire. Results: The cohabitation of parents and adolescents significantly reduced the odds ratio of tobacco smoking, but had a lower effect on reducing drugs use and was not related to alcohol intake. Conclusion: Parental smoking seems to be related only to adolescent smoking. No interaction was found between the two variables in relation to the consumption of any of studied substances.

Key words: smoking, family, alcohol, drugs.
\end{abstract}

Universidade do Vale do Rio dos Sinos (UNISINOS) (Horta RL)

Prontamente - Clínica da Família (Horta RL)

Universidade Federal de Pelotas (UFPel) (Horta BL)

Recebido

07-08-06

Aprovado Universidade Católica de Pelotas (UCPel) (Pinheiro RT)

20-09-06

Projeto original desenvolvido na UCPel com financiamento obtido junto à Fundação de Amparo à Pesquisa do Estado do Rio Grande do Sul (FAPERGS). 


\section{Introdução}

Novos tempos trazem novas configurações familiares e a transformação no exercício dos papéis familiares. Mulheres e homens disputam o mercado de trabalho e estereotipias e papéis definidos em outros tempos perdem espaço (Grzybowski, 2003; Hennigen e Guareschi, 2002; IBGE, 2002). Novas dinâmicas e novos papéis acarretam temores específicos, como ocorreu, por exemplo, com as crianças nascidas de casais que se divorciaram nos primeiros anos de vigência da Lei do Divórcio (Wagner, 2002).

As redes de distribuição de substâncias psicoativas (SPAs), sejam lícitas ou ilícitas, ocupam os espaços de difusão da oferta e da sedução ao consumo e estão atentas aos movimentos sociais, como os fenômenos da inclusão das mulheres no mercado de trabalho e/ou o da expansão da mídia eletrônica (Costa e Silva e Koifman, 1998).

A população adolescente merece ser acompanhada com especial atenção por representar o grupo populacional mais vulnerável à experimentação de álcool, tabaco e outras drogas (Carlini et al., 2002; Giusti et al., 2002; Silva et al., 2003; Soldera et al., 2004).

Parece importante que se identifiquem fatores de proteção e de risco, ou seja, fenômenos que se associam ao do uso de SPA, facilitando ou dificultando sua expansão. Isso pode melhor orientar ações nos campos da prevenção e do tratamento dos problemas decorrentes do uso de SPA, hoje largamente deficientes (Dalgalarrondo et al., 2004; Dalgalarrondo et al., 2005; El-Guebaly, 2005; Selby e Vaccarino, 2005; Uchtenhagen, 2005).

Este artigo pretende avaliar a associação entre o consumo de substâncias psicoativas (bebidas alcoólicas, tabaco e drogas ilícitas) por adolescentes do município de Pelotas (RS), segundo a presença de pai e/ou mãe no domicílio, bem como o hábito de fumar dos pais.

\section{Métodos}

Em estudo realizado em Pelotas (RS), em 2002, a equipe de pesquisas do Programa de Pós-Graduação em Saúde e Comportamento da Universidade Católica de Pelotas (UCPel) entrevistou 960 adolescentes, entre 15 e 18 anos de idade, residentes na zona urbana do município. A amostragem foi realizada, em múltiplos estágios, a partir dos 448 setores censitários da zona urbana. Desses setores, 90 foram sorteados sistematicamente para inclusão no estudo. A seguir selecionou-se, aleatoriamente, um quarteirão de cada setor e sorteou-se uma esquina desse quarteirão para ser o ponto inicial, a partir do qual 86 residências foram visitadas de forma sistemática. Quando necessário, casas de outros quarteirões foram incluídas, escolhidas de forma padronizada, a fim de completar o número de 86 residências. No total, 7.740 domicílios foram visitados pela equipe de pesquisa. Em cada lar sorteado foram entrevistados todos os adolescentes com idades entre 15 e 18 anos completos, depois de obtido 0 consentimento por escrito dos pais ou de outra pessoa adulta responsável (Horta, 2002).

Os adolescentes responderam a um questionário autoaplicado, no qual se buscavam informações sobre comporta- mentos em saúde. Foram selecionados para o exame neste artigo os desfechos:

- uso de tabaco em um período da vida (experimentação) e uso recente de tabaco (pelo menos uma vez por semana no mês que antecedeu as entrevistas);

- consumo de bebidas alcoólicas: foi solicitado que se informasse ter ou não havido consumo de bebidas alcoólicas no mês que antecedeu as entrevistas;

- uso de drogas ilícitas: investigou-se o consumo de qualquer das seguintes drogas na vida: maconha, cocaína e solventes.

Foram tomadas como variáveis independentes coabitação do(a) adolescente com pai e/ou mãe e hábito de fumar de um ou do outro.

Realizaram-se análises univariada e bivariada (teste quiquadrado $\left.\left[\chi^{2}\right]\right)$ e, a seguir, foram calculadas as razões de chances (odds ratio [OR]), com intervalo de confiança (IC) de 95\% para cada um dos desfechos examinados em relação a cada uma das variáveis independentes. Foram consideradas estatisticamente significativas as associações cujo valor de $p$ foi $<0,05$. Essa análise foi ajustada para os seguintes possiveis fatores de confusão: idade, nível socioeconômico e escolaridade materna.

\section{Considerações éticas}

O questionário era auto-aplicado, sem dados de identificação pessoal, e era depositado por quem o respondera, logo após a entrevista, em uma urna lacrada, o que garantia o sigilo das informações. 0 projeto foi aprovado pelo Comitê de Ética em Pesquisa.

\section{Resultados}

$\mathrm{Na}$ amostra estudada, identificaram-se uso continuado de cigarros em 157 sujeitos (16,6\%); uso num período da vida entre 408 pessoas (43\%); uso recente de álcool para 410 indivíduos (43,3\%); uso recente de maconha, cocaína ou solventes entre 86 adolescentes (9\%); e uso na vida de maconha, cocaína ou solventes entre 127 adolescentes (13,8\%).

A razão entre uso num período da vida e uso continuado de cigarros é de 2,6:1, indicando que de cada 2,6 jovens que experimentam cigarros, um permanece em uso continuado, pelo menos na faixa etária estudada.

A análise dos desfechos uso de bebidas alcoólicas, uso de cigarros e consumo de qualquer das drogas ilicitas, todos no mês que antecedeu à entrevista, segundo a coabitação ou não com pai e/ou mãe, leva aos dados apresentados na Tabela 1. O comportamento dos desfechos não foi homogêneo entre as substâncias em estudo.

O risco de consumo de bebidas alcoólicas parece não estar associado à presença ou não de pai e/ou mãe nos domicílios. Ao considerar como referência a coabitação do(a) adolescente, tanto com o pai quanto com a mãe, a OR para coabitação com apenas pai ou mãe é de 1,23 (IC 95\%: 0,83 a 1,83 ), e para não-coabitação com nenhum deles é de 1,11 (IC 95\%: 0,65 a 1,87).

A OR de uso de cigarros parece aumentar quando pai e/ou mãe não estão presentes nos domicílios. Ao adotar como 
referência a coabitação do(a) adolescente, tanto com o pai quanto com a mãe, a OR para coabitação com apenas pai ou mãe é de 2,17 (IC 95\%: 1,38 a 3,4), e para não-coabitação é de 2,52 (IC 95\%: 1,3 a 4,88).

A chance de surgir o relato de consumo de maconha, cocaína ou solventes parece não apresentar variação quanto à presença de pai e mãe nos domicílios, ou de apenas um dos dois. A diferença mais significativa se expressou quando o(a) adolescente não coabitava com nenhum dos dois. Tendo como referência a coabitação do(a) adolescente, tanto com o pai quanto a com mãe, a OR para coabitação com apenas pai ou mãe é de 1,06 (IC: 0,63 a 1,79), e para não-coabitação é de 2,01 (IC: 0,99 a 4,11).

\section{Tabela 1. Riscos de ocorrência dos desfechos segundo a configuração do domicílio}

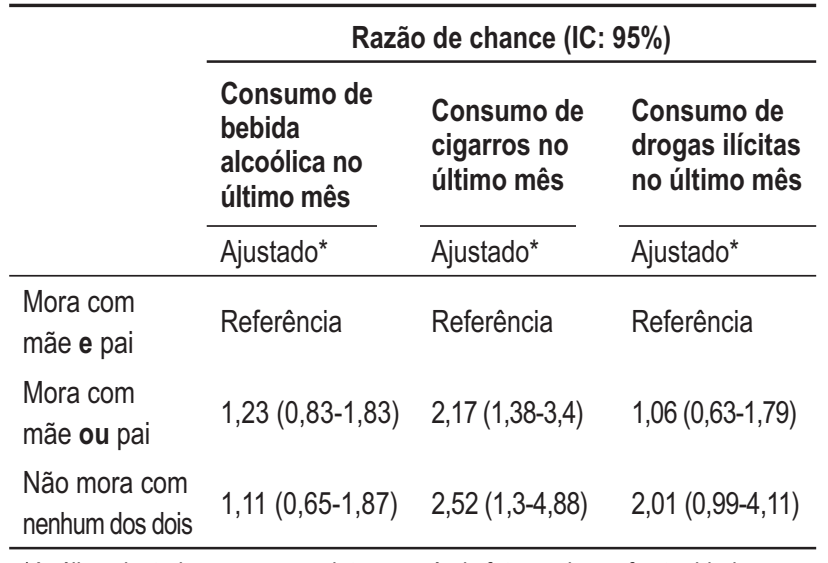

*Análise ajustada para os seguintes possíveis fatores de confusão: idade, nível socioeconômico e escolaridade materna. IC: intervalo de confiança.

A análise dos mesmos desfechos, segundo o hábito de fumar ou não de pai e/ou mãe do(a) adolescente, leva aos dados apresentados na Tabela 2.

Para bebidas alcoólicas parece não ter havido associação significativa entre o consumo da população investigada e o tabagismo paterno ou materno. Há maior risco de ocorrência do comportamento consumo de substâncias ilícitas quando os sujeitos têm pais e mães fumantes, e aumento mais discreto quando apenas o pai ou a mãe são fumantes. 0 efeito mais significativo se apresenta quando se analisa o risco de ocorrência de tabagismo em relação ao hábito de fumar de pais ou mães.

Ao considerar como referência a condição de pai e mãe não-fumantes, a chance de ocorrência de tabagismo entre adolescentes com pai ou mãe fumantes é de 1,99 (IC 95\%: 1,28 a 3,09), e para ambos fumantes é de 3,92 (IC 95\%: 2,29 a 6,71$)$ vezes maior em relação ao grupo de comparação.

A chance de surgir o relato de consumo de maconha, cocaína ou solventes parece acompanhar a variação do risco de ocorrência de tabagismo, porém com menor amplitude. Tendo como referência a condição de pai e mãe não-fumantes, a chance de ocorrência de consumo de qualquer das drogas ilícitas estudadas, quando pai ou mãe são fumantes, é de 1,87 (IC 95\%: 1,15 a 3,05), e para ambos fumantes é de 2,04 (IC 95\%: 0,97 a 4,29 ) (Tabela 2).
Tabela 2. Riscos de ocorrência dos desfechos segundo o tabagismo de pais e de mães

\begin{tabular}{|c|c|c|c|}
\hline & \multicolumn{3}{|c|}{ Razão de chance (IC: 95\%) } \\
\hline & $\begin{array}{l}\text { Consumo de } \\
\text { bebida } \\
\text { alcoólica no } \\
\text { último mês }\end{array}$ & $\begin{array}{l}\text { Consumo de } \\
\text { cigarros no } \\
\text { último mês }\end{array}$ & $\begin{array}{l}\text { Consumo de } \\
\text { drogas ilícitas } \\
\text { no último mês }\end{array}$ \\
\hline & Ajustado* & Ajustado* & Ajustado* \\
\hline $\begin{array}{l}\text { Pai e mãe } \\
\text { não-fumantes }\end{array}$ & Referência & Referência & Referência \\
\hline $\begin{array}{l}\text { Pai ou mãe } \\
\text { fumante }\end{array}$ & $1,11(0,77-1,59)$ & $1,99(1,28-3,09)$ & $1,87(1,15-3,05)$ \\
\hline $\begin{array}{l}\text { Pai e mãe } \\
\text { fumantes }\end{array}$ & $1,32(0,77-2,25)$ & $3,92(2,29-6,71)$ & $2,04(0,97-4,29)$ \\
\hline
\end{tabular}

*Análise ajustada para os seguintes possíveis fatores de confusão: idade, nível socioeconômico e escolaridade materna. IC: intervalo de confiança.

Não foi observada interação entre o hábito de fumar de pais e/ou mães e coabitação com pais e/ou mães.

\section{Discussão}

Neste estudo verificou-se que a presença de pai, mãe ou ambos no domicílio parece ter efeito protetor contra o uso de tabaco e, possivelmente, tenha o mesmo efeito quanto às drogas ilícitas, conquanto não houve associação com o uso de álcool. A baixa ocorrência do desfecho uso de drogas ilícitas faz com que 0 estudo apresente menor poder estatístico na avaliação dos fatores associados a esse desfecho.

No tocante ao tabagismo dos pais, percebe-se um risco significativamente elevado de tabagismo e de uso de drogas ilícitas por adolescentes quando tanto o pai quanto a mãe são fumantes. $\mathrm{O}$ risco se reduz quando apenas o pai ou a mãe fumam. $\mathrm{O}$ hábito de fumar de pais e mães parece não ter tido qualquer relação com o uso de álcool por adolescentes neste estudo.

A família e a cultura transmitem sistemas de crenças e expectativas sobre os papéis sociais, sobre o modo de vida de homens e mulheres, sobre as relações entre os seres humanos e também sobre usos e costumes, como em relação às SPAs.

Ao analisarmos o papel da família na transmissão de pautas de comportamento, devemos considerar o permanente atravessamento da cultura contemporânea e seus valores oscilantes. Parece que estamos vivendo o início de uma nova era, com sua dinâmica de mutações globais, que fragmenta os vínculos de identidade e gera isolamentos (Morin, 1997). Constatar o efeito protetor da presença parental pode servir para reduzir os temores ligados a idéias de fragmentação ou desconstituição dos grupos familiares.

Até algumas décadas atrás, a família poderia ser facilmente compreendida como um grupo de pessoas que interagem em um todo funcional, um sistema que consistia em uma rede completa de parentesco de pelo menos três gerações, em que as interações tendiam a ser recíprocas e padronizadas. Na contemporaneidade, esse conceito de família precisa ser ampliado para incluir as novas constelações familiares como casamentos sem filhos, casamentos que agrupam crianças de diferentes famílias, 
lares uniparentais, entre outros. Já se identifica na literatura número expressivo de produções em torno do tema "famílias reconstituídas", definidas pela sobreposição de constituições e rupturas de uniões conjugais entre seus membros (Minuchin, 1982; Falicov, 1991; Gonzáles, 1994; Feres-Carneiro, 1999; Wagner, 2002).

A família contemporânea está imersa nas revoluções da informação e da tecnologia, que afetam diariamente a vida dos indivíduos e dos grupos, por meio da televisão e do computador, e invadem o mundo perceptivo e os sistemas de crenças, com padrões de comportamento e apelo ao consumo (Horta, 2003).

Sabemos que a admiração filial induz a criança a imitar suas figuras parentais. Num espectro ampliado de estímulos e referências, poder-se-ia esperar que a presença física de pais e mães no domicílio tivesse menor efeito. Este estudo evidencia 0 efeito protetor que se exerce no presente.

A correlação estabelecida entre o hábito de fumar de adolescentes e o de pais e/ou mães incrementa a expectativa de que esse comportamento possa ser aprendido em casa, mais do que no espaço público. Seria, pelo menos em parte, uma tendência à repetição de comportamentos ou vivências. Faz parte dos ciclos da aprendizagem informal. Por isso mesmo a formação de consumidores e consumidoras de SPAs representa a formação de algumas gerações de consumidoras e consumidores desse produto. A indústria tabagista sabe disso (Costa e Silva e Koifman, 1998).

Os dados apresentados nas Tabelas 1 e 2 não permitem sustentar ou propor hipóteses de esvaziamento das funções parentais na pós-modernidade, nem idéias de culpabilização de mães e pais pelo uso de SPAs que seus filhos ou filhas venham a fazer. O padrão de interação entre as variáveis estudadas e os desfechos escolhidos em relação ao consumo de SPAs pelos adolescentes não é uniforme entre os grupos de substâncias. As famílias parecem manter, na atualidade, suas funções e as dificuldades inerentes aos papéis de proteção, preparo e iniciação das populações mais jovens em suas diversas configurações e com seus hábitos e costumes (Horta, 2003; Wagner, 2002).

Considerou-se a importância do hábito de fumar de modo independente da configuração familiar no domicílio para que se contemplasse também o exemplo e outros meios pelos quais os hábitos de pais e mães venham a ser reproduzidos ou evitados por filhas e filhos, sejam eles avaliados como negativos ou positivos. Assim, a influência de pai e/ou mãe não se restringe necessariamente à moradia conjunta.

Ao considerar a moradia conjunta, a exposição ambiental ao fumo sem que a própria pessoa exerça a atitude de fumar deve ser lembrada aqui. O fumo passivo seria uma forma adicional de reprodução do comportamento entre gerações.
Carvalho e Pereira (2002) estudaram 1.104 crianças menores de 5 anos de idade. A morbidade respiratória foi maior entre aquelas expostas ao fumo ambiental do que entre as não-expostas (82\% vs. 74\%). A análise de regressão logística mostrou que as queixas referentes ao trato respiratório inferior se associaram com maior probabilidade a tabagismos materno e paterno, com chances quase iguais e, só depois, ao mofo e aos antecedentes de doenças respiratórias. Isso corresponde a dizer que crianças expostas ao hábito de fumar de pessoas adultas já são fumantes na modalidade passiva. Aqui se trata não apenas da transmissão de pauta de comportamentos, mas da iniciação propriamente dita no consumo de tabaco.

Os dados deste estudo permitem descortinar a complexidade da determinação de comportamentos relacionados às SPAs por adolescentes, não se podendo estabelecer uma linearidade causal. Os efeitos do comportamento e da presença de pais e mães sobre o desenvolvimento e o comportamento de suas proles também parecem relacionados a uma trama complexa de variáveis, em sua maioria não examinada neste artigo. Essa complexidade pode estar associada à flexibilidade dos sistemas familiares.

\section{Conclusão}

A primeira das considerações finais deste estudo é ressaltar a importância de estarmos atentos para que dados semelhantes não ganhem significado distorcido. Os estudos apresentados mostram a força que o padrão familiar de consumo de substâncias possui na perpetuação do uso de tabaco e de drogas, mas também aponta a família em seu poder como transmissora de comportamentos, quer isso contribua para a redução e/ou para a expansão do hábito de fumar.

Os estudos mostram a necessidade de estarmos, como profissionais, com a atenção voltada para as mudanças sócio-históricas nas quais estamos inseridos, sempre dedicados ao exame de aspectos qualitativos e subjetivos dos fenômenos, para que possamos nos deparar com contradições, como as que colocam pais e mães, simultaneamente, nos dois pólos da proteção e da exposição ao risco de consumo de SPAs por suas proles.

A constatação de que o tabagismo paterno ou materno aumenta a chance de a sua prole desenvolver o hábito de fumar se agrega ao conjunto de investigações que buscam demonstrar o fenômeno do "fumo passivo".

Padrões de configurações familiar e dos domicílios parecem variáveis relevantes no planejamento de ações de prevenção ao uso de droga, incentivo à vida saudável e, também, para a clínica dos problemas eventualmente decorrentes do uso de SPAs. 


\section{Referências}

Carlini EA et al. (org.). I Levantamento Domiciliar Sobre o Uso de Drogas Psicotrópicas no Brasil. São Paulo: Cebrid; 2002.

Carvalho LMT, Pereira E. Morbidade respiratória em crianças fumantes passivas. J Pneumol, 28(1): 8-14, 2002.

Costa e Silva VL, Koifman S. Smoking in Latin America: a major public health problem. Cad Saúde Pública, 14(suppl 3): 109-15, 1998. Available in: <http://www.scielo.br/>. Access in: 11 nov. 2005.

Dalgalarrondo P et al. Religião e uso de drogas por adolescentes. Rev Bras Psiquiatr, 26(2): 82-90, 2004.

Dalgalarrondo $P$ et al. Jovens pentecostais e espíritas em comparação a católicos: uso de álcool e drogas e saúde mental. J Bras Psiquiatr, 54(3): 182-90, 2005.

El-Guebaly N. Don't drink and drive: the successful message of mothers against drunk driving (Madd). World Psychiatry, 4(1): 35-6, 2005.

Falicov C. (org). Transiciones de la família. Buenos Aires: Amorrortu Editores; 1991.

Feres-Carneiro T. (org.). Casal e família: entre a tradição e a transformação. Rio de Janeiro: NAU Editora; 1999.

Giusti J, Sañudo A, Scivoletto S. Differences in the pattern of drug use between male and female adolescents in treatment. Rev Bras Psiquiatr, 24(2): 80-2, 2002.

Gonzáles JAR. Manual de Orientación y Terapia Familiar. Madrid: Centro de Estudios Ramón Areces; 1994.

Grzybowski LS. Famílias monoparentais: reflexo da pós-modernidade? In: Guareschi P. et al. (org.). Psicologia em questão: reflexões sobre a contemporaneidade. Porto Alegre: Edipucrs; 2003.

Hennigen I, Guareschi NMF. A paternidade na contemporaneidade: um estudo de mídia sob a perspectiva dos estudos culturais. Psicologia \& Sociedade, 14(1): 44-68, 2002.

Horta BL. Prevalência de comportamentos de saúde em adolescentes na cidade de Pelotas (RS). Relatório de Pesquisa. CNPq; 2002.

Horta RL. Famílias e drogas na contemporaneidade. In: Guareschi $P$ et al. (org.) Psicologia: questões sobre a contemporaneidade. Porto Alegre: Edipucrs; 2003.

IBGE (Instituto Brasileiro de Geografia e Estatística). Perfil das mulheres responsáveis pelos domicílios no Brasil - 2000: estudos e pesquisas: informação demográfica e socioeconômica. 8 ed. Rio de Janeiro: IBGE; 2002.

Minuchin S. Famílias: funcionamento e tratamento. Porto Alegre: Artes Médicas; 1982.

Morin E. Introducción al pensamiento complejo. Barcelona: Gedisa, 1997.

Selby P, Vaccarino F. Substance abuse prevention: practical strategies for psychiatrists in the 21 ${ }^{\text {st }}$ century. World Psychiatry, 4(1): 32-3, 2005.

Silva V. et al. Brazilian study on substance misuse in adolescents: associated factors and adherence to treatment. Rev Bras Psiquiatr, 25(3): 133-8, 2003.

Soldera M et al. Uso pesado de álcool por estudantes dos ensinos fundamental e médio de escolas centrais e periféricas de Campinas (SP): prevalência e fatores associados. Rev Bras Psiquiatr, 26(3): 174-9, 2004.

Uchtenhagen A. How effective is substance abuse prevention? World Psychiatry, 4(1): 33, 2005.

Wagner A (org.). Família em cena: tramas, dramas e transformações. Petrópolis: Editora Vozes; 2002. 